\title{
Order Independent Homotopic Thinning
}

\author{
Vincent Ranwez ${ }^{1}$ and Pierre Soille ${ }^{2 \star}$ \\ 1 Dpt. Informatique Fondamentale et Applications \\ LIRMM, 161 rue ADA, F-34392 Montpellier, FRANCE \\ ranwez@lirmm.fr, \\ http://www.lirmm.fr/ ${ }^{\sim}$ ranwez \\ 2 Silsoe Research Institute, Image Analysis Group, Wrest Park, Silsoe \\ Bedfordshire MK45 4HS, U.K. \\ pierre.soille@bbsrc.ac.uk
}

\begin{abstract}
This article introduces an order independent homotopic thinning for binary pictures. This thinning has the following properties: it does not use homotopic structuring elements, it is independent of the order in which pixels are processed, it is invariant through $\pi / 2$ rotations, and it takes into account global characteristics of the image through Ronse's criterion of strong 8-deletability. An algorithm implementing this new thinning is presented and applied to the skeletonisation of binary patterns.
\end{abstract}

\section{Introduction}

Image homotopic thinning is a usual pre-processing transformation in pattern recognition. Its aim is to provide a compact representation of the image while preserving its topological properties. The present paper introduces a homotopic thinning algorithm independent of the order in which pixels are processed.

This paper is organised as follows. Basic definitions and notations are provided in Section 2 Section 3 introduces the notion of independent simple pixels and proposes a characterisation of such pixels. Section 4 concerns the implementation of the resulting thinning algorithm and the study of its properties. Before concluding, section 5 shows that order independent thinnings can be used for skeletonising binary patterns.

\section{Definitions and notations}

In this section we consider discrete binary images in the square grid. Some basic concepts like discrete image, discrete distance, and discrete neighbourhood are assumed to be known by the reader. Rigourous definitions of these concepts as well as a good introduction to digital topology can be found in KR89 and CM91. In the following definitions and notations, $X$ equals either 4 or 8 .

* This work has been initiated while the authors were with the LGI2P, Ecole des Mines d'Alès \& EERIE (Nîmes). 


\subsection{Neighbourhood and connectivity}

We denote by $d_{X}$ the $X$-connected distance, by $N_{X}(P)$ the set of $X$-connected neighbours of a pixel $P$, and by $\bar{N}_{X}(P)$ the set $N_{X}(P) \cup P$. The set of $X$ connected neighbour pixels of $P$ with the value $b(b \in\{0,1\})$ is denoted by $N_{X}^{b}(P)$ and is defined as follows: $N_{X}^{b}(P)=\cup_{i}\left\{P_{i} \in N_{X}(P) \mid \operatorname{Im}\left(P_{i}\right)=b\right\}$. Similarly, $\bar{N}_{X}^{b}(P)=N_{X}^{b}(P) \cup P$.

These definitions are easily extended to an arbitrary set $S$ of pixels: $\bar{N}_{X}(S)=$ $\cup_{P \in S} \bar{N}_{X}(P), N_{X}(S)=\bar{N}_{X}(S) \backslash S, \bar{N}_{X}^{b}(S)=\cup_{P \in S} \bar{N}_{X}^{b}(P)$, and $N_{X}^{b}(S)=$ $\bar{N}_{X}^{b}(S) \backslash S$.

We call $X$-path a sequence of pixels such that each pixel of the sequence is a $X$-connected neighbour of the next one. The first and the last pixels of this sequence are called the extremities of the path. A $X^{b}$-path is a $X$-path whose pixels have all the value $b$.

In an image $I m$, two pixels $P_{0}$ and $P_{n}$ are $X^{b}$-connected iff there exists a $X^{b}$-path between $P_{0}$ and $P_{n}$. The relation to be $X^{b}$-connected is an equivalence relation. The corresponding equivalence classes define the $X^{b}$-connected components of pixels. They are denoted by $C C_{X}^{b}$. Finally, the $X$-connected component containing $P$ will be denoted by $\overline{C C_{X}}(P)$ and consequently $C C_{X}(P)=$ $\overline{C C_{X}}(P) \backslash P$.

\subsection{Homotopy}

We will now place our study within the common practical framework Ros70 by using the 4-connectivity for the background (pixels with the value 0) and the 8-connectivity for the foreground (pixels with the value 1). We also assume that the pixels outside of the image definition domain are background pixels. By doing so, we make sure that there exists a $C C_{4}^{0}$, noted $S_{\text {outside, }}$ which is the supremum of the embedding relation $\sqsubseteq$ detailed hereafter.

Let $S_{1}$ be a $C C_{8}^{1}, S_{0}$ a $C C_{4}^{0}$, and $S$ either a $C C_{8}^{1}$ or a $C C_{4}^{0}$. Then, $S_{1} \sqsubseteq S$ iff every 4-path linking a pixel of $S_{1}$ to a pixel of $S_{\text {outside }}$ contains at least one pixel of $S$. Similarly, $S_{0} \subseteq S$ iff every 4-path linking a pixel of $S_{0}$ to a pixel of $S_{\text {outside }}$ contain at least one pixel of $S$.

The adjacency tree Bun69] or homotopy tree Ser82] of a binary image is a graph whose vertices represent the background and foreground connected components, whose root is $S_{\text {outside }}$ and where the ascendents of a vertex $V$ are the vertices corresponding to the connected components embedding $V$ (i.e., two vertices are joined by an edge if and only if the corresponding embedded connected components are adjacent to each other KR89]). Two 2-D binary images $I m_{1}$ and $\mathrm{Im}_{2}$ are homotopic if and only if they have the same homotopy tree Ser82.

Finally, a pixel is called deletable Ros70] or simple [Kon95 if and only if the image obtained by removing this pixel (i.e. changing its value from 1 to 0 ) is homotopic to the original image. We call simpleness property the property of being simple. Depending on whether a pixel is simple or not, the simpleness test will return true or false. Rosenfeld's characterisation of a simple pixel [Ros70] 
provides us with a practical definition for implementing the simpleness test. Indeed, he has shown that a pixel $P$ of a 2-D binary image $I m$ is simple if and only if the two following conditions hold:

1. The set of 8-connected foreground neighbours of $P, N_{8}^{1}(P)$, is nonempty and 8-connected;

2. The set of 4-connected background neighbours of $P, N_{4}^{0}(P)$, is nonempty.

\section{Characterisation of order independent simple pixels}

\subsection{The notion of independence}

A trivial order dependent homotopic thinning algorithm applied to a binary image $I m$ is

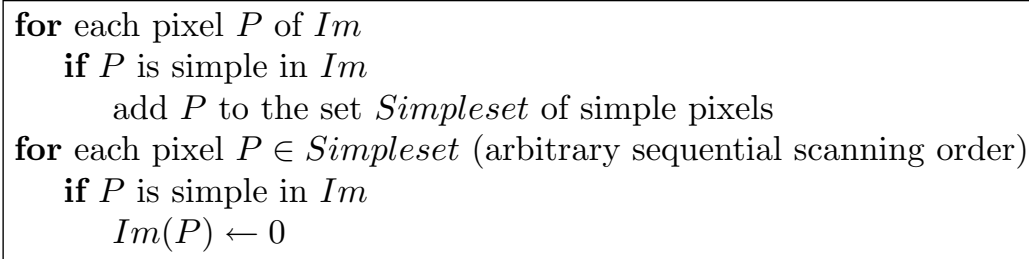

This algorithm will be referred to as Algo1. At the end of Algo1, the input image $I m$ contains a thinned image which is homotopic to the original image. Unfortunately, the resulting image depends on the scanning order of the set of simple pixels. An order-independent independent homotopic thinning algorithm can be obtained by setting to 0 those pixels of the original image which are set to 0 by Algo1 whatever the considered scanning order (i.e., for all possible scanning orders). We call these pixels the order independent simple pixels and they are said to be independent.

\subsection{Independence with regard to a single pixel}

We will first consider the case where a simple pixel $P$ has only one simple pixel $Q$ in its neighbourhood. In this particular case we will say that $P$ is (in)dependent of $Q$ in the sense that the removal of $P$ is (in)dependent of the order in which the simple pixels are computed. In such a case the only two possible configurations of the neighbourhood of $P$ are: (i) the one with $Q=1$, obtained by all scanning orders, where $P$ is processed first, and (ii) the one with $Q=0$ obtained by all scanning orders where $Q$ is the first pixel processed.

Lemma 1. A simple pixel $P$ is dependent of a simple pixel $Q$ if they have one of the following configurations (plus their rotations through $\pi / 2$ ): 


\begin{tabular}{|c|c|}
\hline 0 & $\mathrm{Q}$ \\
\hline $\mathrm{P}$ & 0 \\
\hline
\end{tabular}

\begin{tabular}{|c|c|}
\hline 0 & 0 \\
\hline $\mathrm{P}$ & $\mathrm{Q}$ \\
\hline 0 & 0 \\
\hline
\end{tabular}

\begin{tabular}{|c|c|}
\hline 1 & $*$ \\
\hline $\mathrm{P}$ & $\mathrm{Q}$ \\
\hline 1 & $*$ \\
\hline
\end{tabular}

\begin{tabular}{|c|c|}
\hline 1 & $*$ \\
\hline $\mathrm{P}$ & $\mathrm{Q}$ \\
\hline$*$ & 1 \\
\hline
\end{tabular}

\begin{tabular}{|c|c|}
\hline$*$ & 1 \\
\hline $\mathrm{P}$ & $\mathrm{Q}$ \\
\hline$*$ & 1 \\
\hline
\end{tabular}

\begin{tabular}{|c|c|}
\hline$*$ & 1 \\
\hline $\mathrm{P}$ & $\mathrm{Q}$ \\
\hline 1 & $*$ \\
\hline
\end{tabular}

$*=0$ or 1 .

Proof. We have to show that the result of the simpleness test of $P$ will be false if $Q$ has the value 0 .

- For the first two configurations, $P$ is simple, this implies that $N_{8}^{1}(P)$ is 8connected. Therefore, $N_{8}^{1}(P)$ contains a single pixel $Q$ and if $Q$ is set to 0 , $N_{8}^{1}(P)$ will be empty. Consequently, $P$ will no more satisfy the first Rosenfeld test.

- For the four other configurations, $P$ is simple, this implies that $N_{4}^{0}(P)$ is nonempty and therefore if $Q$ is set to $0, N_{8}^{1}(P)$ will no longer be 8-connected. Again it follows that $P$ will no more satisfy the first Rosenfeld test.

Theorem 1. A simple pixel $P$ is independent of a simple pixel $Q$ iff they have one of the following configurations (plus their rotations through $\pi / 2$ ):

\begin{tabular}{|c|c|}
\hline$*$ & $\mathrm{Q}$ \\
\hline $\mathrm{P}$ & 1 \\
\hline
\end{tabular}

\begin{tabular}{|l|l|}
\hline 1 & $\mathrm{Q}$ \\
\hline $\mathrm{P}$ & $*$ \\
\hline
\end{tabular}

$*=0$ or 1 .

Proof.

- From right to left:

- First Rosenfeld test: in these configurations, $P$ has an 8-neighbour with the value 1 different of $Q$ and $Q$ plays no role in the connectivity of $N_{8}^{1}(P)$.

- Second Rosenfeld test: $P$ is simple so $N_{4}^{0}(P)$ is nonempty and this will not change by setting $Q$ to 0 .

This demonstrates that the simpleness test of $P$ is independent of the value of $Q$ : it returns true whatever the value of $Q$.

- From left to right: if $P$ and $Q$ are in an other configuration they are in one of the configuration of lemma 1 and therefore $P$ is dependent of $Q$. 


\subsection{Strict independence}

Let us now consider the general case where there are $n$ simple pixels $Q_{1}, \ldots, Q_{n}$ in the neighbourhood of $P$. We denote by $Q_{i}$ any one of these simple pixels.

If $P$ is dependent of $Q_{i}, P$ is not removed for sequences starting by $Q_{i}$. This implies that for being independent, $P$ must be independent of all its simple neighbours.

By assuming that the ' 1 ' pixels shown in the configurations of theorem 2 are all nonsimple (i.e., they do not match any $Q_{i}$ ), we define a strict independence between $P$ and its simple neighbours in the sense that the simpleness test of $P$ is independent of the value of its simple neighbours. This guarantees that, if the ' 1 ' pixels in the configurations shown in theorem 2 are nonsimple pixels, $P$ will always be removed by Algo1.

Now, the only configurations for which we have not yet a result are those where $P$ is independent of all the $Q_{i}$ but there exists at least one of the $Q_{i}$ noted $Q_{s}$ such that $P$ is independent of $Q_{s}$ but not strictly. In such a case, the simpleness test of $P$ depends on the value of its simple neighbours. Nevertheless, since these pixels are themselves interdependent, all combinations are not possible. This restricts the neighbourhood configurations of $P$. All configurations where $P$ is independent of $Q_{s}$ but not strictly are the configurations of independence such that the only possible matching between these configurations and the neighbourhood of $P$ implies that at least a ' 1 ' pixel matches with a pixel of $\left\{Q_{1}, \ldots, Q_{n}\right\} \backslash Q_{s}$. These configurations are the following (plus their rotations through $\pi / 2)$ :

\begin{tabular}{|c|c|}
\hline 0 & Qs \\
\hline $\mathrm{P}$ & $\mathrm{Q} 1$ \\
\hline
\end{tabular}

\begin{tabular}{|c|c|}
\hline Q1 & Qs \\
\hline P & Q2 \\
\hline
\end{tabular}

\begin{tabular}{|c|c|}
\hline 0 & $\mathrm{Q} 1$ \\
\hline $\mathrm{P}$ & $\mathrm{Qs}$ \\
\hline 0 & 0 \\
\hline
\end{tabular}

\begin{tabular}{|c|c|}
\hline Q1 & Q2 \\
\hline P & Qs \\
\hline 0 & 0 \\
\hline
\end{tabular}

\begin{tabular}{|c|c|}
\hline Q1 & 0 \\
\hline P & Qs \\
\hline 0 & 0 \\
\hline
\end{tabular}

In those configurations $P, Q_{s}, Q_{1}$, and $Q_{2}$ are simple pixels. We must keep in mind that in Algo1 the first processed simple pixel has the neighbourhood found in the original image. Therefore, it is always simple and will be removed. In addition, the simple pixels processed after $P$ have the value 1 when processing $P$. Assuming that $P$ is independent, we find the following restrictions (we denote by sequences $(P, R)$ the set of scanning sequences of Algo1 having $P$ as first pixel and $R$ as second):

- configuration 1: $P$ must be simple for sequences $\left(Q_{1}, P\right)$ this implies that all the not displayed neighbours of $P$ must have the value 0 . Otherwise they would not be connected with $Q_{s}$. Therefore the second Rosenfeld test would fail and $P$ would not be removed.

- configuration 2: as $P$ is simple, it has a 4-connected neighbour with the value 0 . Repeating twice the same reasoning than in configuration 1 for 
sequences $\left(Q_{1}, P\right)$ and for sequences $\left(Q_{2}, P\right)$, we deduce that all the not displayed neighbours of $P$ have the value 0 .

- configuration 3: the fact that $P$ is simple implies that its not displayed neighbours have the value 0 .

- configuration 4: sequences $\left(Q_{1}, P\right)$ implies that the not displayed neighbours of $P$ have the value 0 .

- configuration 5: sequences $\left(Q_{1}, P\right)$ implies that the not displayed 8-neighbours of $P$ have the value 0 . Consequently, the fact that $Q_{s}$ and $Q_{1}$ are simple implies that their not displayed neighbours have also the value 0 . Therefore, for sequences $\left(Q_{s}, Q_{1}, P\right), Q_{s}$ is removed because it is simple. This does not change the fact that $Q_{1}$ is simple and thus $Q_{1}$ is also removed. Hence, $N_{8}^{1}(P)$ is empty and therefore $P$ is not removed.

\subsection{Ronse's theorem}

Following the restrictions detailed in the previous section, the only configurations that still need to be considered are (plus their rotations through $\pi / 2$ ):

\begin{tabular}{|c|c|c|}
\hline 0 & 0 & Qs \\
\hline 0 & $P$ & Q1 \\
\hline 0 & 0 & 0 \\
\hline
\end{tabular}

\begin{tabular}{|c|c|c|}
\hline 0 & $\mathrm{Q} 1$ & $\mathrm{Qs}$ \\
\hline 0 & $\mathrm{P}$ & $\mathrm{Q} 2$ \\
\hline 0 & 0 & 0 \\
\hline
\end{tabular}

\begin{tabular}{|c|c|c|}
\hline 0 & 0 & $\mathrm{Q} 1$ \\
\hline 0 & $\mathrm{P}$ & $\mathrm{Qs}$ \\
\hline 0 & 0 & 0 \\
\hline
\end{tabular}

\begin{tabular}{|c|c|c|}
\hline 0 & $\mathrm{Q} 1$ & $\mathrm{Q} 2$ \\
\hline 0 & $\mathrm{P}$ & $\mathrm{Qs}$ \\
\hline 0 & 0 & 0 \\
\hline
\end{tabular}

In these configurations it is obvious that the only cases where $P$ is not simple are the cases where all the $Q_{i}$ have the value 0 . Moreover, every 8-path linking $P$ to $C C_{8}^{1}(P) \backslash\left\{Q_{1}, \ldots, Q_{n}\right\}$ contains at least one $Q_{i}$ pixel. Therefore, to allow the last treated $Q_{i}$ to be removed, all other pixels of $C C_{8}^{1}(P)$ must have already been removed. Otherwise, the last $Q_{i}$ pixel is not simple and will not be removed. This implies that $C C_{8}^{1}(P)$ contains only simple pixels. In conclusion, the two conditions of the existence of a scanning order in which $P$ is not removed by Algo1 are the following: (i) $C C_{8}^{1}(P)$ contains only simple pixels and (ii) it exists a scanning order of simple pixels such that all pixels of $C C_{8}^{1}(P)$ are removed before $P$. The first condition is easy to test and the second one can be answered by Ronse's characterisation of 8-strongly deletable set for a nonempty set of 8-connected pixels:

Theorem 2. Ron86 let Im be an image, let $D$ be a set of 8-connected foreground pixels of Im, let $t=|D|$ and Im' be the image obtain from Im by removing all the pixels of the set $D$. The following statements are equivalent:

1. $D$ is strongly 8-deletable from Im

2. D corresponds to Ronse's characterisation of a 8-deletable set

- the set $N_{8}^{1}(D)$ belongs to the same $C C_{8}^{1}$ of $\mathrm{Im}^{\prime}$

- given $D_{1}, \ldots, D_{n}$ the $C C_{4}^{1}$ of $D$, for all $i$ in $\{1, \ldots, n\}$, the set $N_{4}^{0}\left(D_{i}\right)$ belongs to the same $C C_{4}^{0}$ of $I m$.

3. we can label the pixels of $D, Q_{1}, \ldots, Q_{t}$, in such a way that each $Q_{i}$ is simple in $\operatorname{Im} \backslash\left\{Q_{j} \mid j<i\right\}$. 


\section{The order independent thinning algorithm}

\subsection{Characterisation of order independent simple pixels}

We will sum up the previous section to show that in all cases we have either found a sequence where $P$ is not removed or it will be removed for all possible sequences. Indeed, given a simple pixel $P$ :

if $P$ is dependent of one of its simple neighbours,

then we have found an order where $P$ is not removed. else

if $P$ is strictly independent of all its simple neighbours,

then we have shown that $P$ will always be removed.

else

if $P$ and $Q$ are not in one of the configurations shown in section

3.4 ,

then there exists an order where $P$ is not removed.

else

if $C C_{8}^{1}(P)$ contains only simple pixels and is 8-deletable,

then Ronse's theorem guarantees that there is an order where

$P$ is not removed.

else we have proved that $P$ will always be removed.

\subsection{Useful properties for the implementation}

- The simpleness is a local property. Thus, when iterating order independent thinnings, we can restrict our research for new simple pixels to the neighbourhood of the simple pixels deleted at the previous iteration.

- Rather than directly considering all configurations and their rotations, we propose to summarise them with the following rules:

1. $P$ is independent of $Q$ if and only if:

- there is another foreground pixel within the intersection of their 8neighbourhood,

- when $P$ and $Q$ are 4 -connected, there is a $4^{0}$-path between them within the intersection of their 8-neighbourhood.

2. $P$ is strictly independent of $Q$ if and only if:

- $P$ is independent of $Q$,

- and moreover there is within the intersection of their 8-neighbourhood a nonsimple foreground pixel.

3. The configurations requiring to test the 8-deletability of a set are those where

- $P$ is (not strictly) independent of $Q$,

- and all neighbours of $P$ which are not neighbours of $Q$ have the value $0\left(\forall P^{\prime} \in N_{8}(P) \backslash N_{8}(Q), \operatorname{Im}\left(P^{\prime}\right)=0\right)$. 
- An important result is provided by a Ronse's lemma 3.1 in [Ron86]: let Im be an image, $D$ a set of 8-connected foreground pixels of $I m, I m^{\prime}=I m \backslash D$, $D^{\prime} \subseteq D$, and $D^{\prime \prime}=D \backslash D^{\prime}$. Then if $D$ is 8-deletable from $I m$ and $D^{\prime}$ is 8-deletable from $\mathrm{Im}$, then $D^{\prime \prime}$ is 8-deletable in $\mathrm{Im}^{\prime}$.

This guarantees that, if a pixel is not independently simple because it is connected to a 8-deletable set, the deletion of simple pixels cannot change this fact. Hence, for each pixel $P$, we test the 8-deletability at most once because either $P$ is independently simple (and is removed) or it is not (and will never be).

\subsection{The algorithm}

In its basic form, the order independent thinning algorithm iterated until idempotence is as follows:

while pixels are deleted

detect the simple pixels

delete the independent simple pixels.

An optimal implementation is out of the scope of this paper. Nevertheless the previous section already provides an implementation having a complexity of $O\left(n^{2}\right)$ where $n$ is the number of pixels of the input image. Indeed, we test each pixel only when its neighbourhood changes, i.e., at most 8 times. The 8deletability of a set can be done in $O(n)$ and is done at most once per pixel. The other tests are local, so finally the complexity of the whole algorithm is $O\left(n^{2}\right)$.

\subsection{Properties of the order independent thinning algorithm}

This thinning algorithm has the following properties:

1. it does not use structuring homotopic elements,

2. it is independent of the order in which the pixels are processed,

3 . it provides a result invariant through $\pi / 2$ rotations,

4. it is naturally adapted to a parallel implementation,

5. it has a polynomial implementation in $O\left(n^{2}\right)$ where $n$ is the number of pixels of the input image,

6. it takes into account global characteristics of the image through Ronse's theorem.

By definition, our algorithm can be viewed as a parallel thinning algorithm. Thus the notion of 8-Minimal Non Deletable sets introduced by [Ron88] could also be used as an alternative to prove its correctness.

\section{The order independent thinning skeleton}

A common way to define a skeleton through homotopic thinning is to perform sequential homotopic thinnings until idempotence [Lan78|Ser82]. If the thinning 


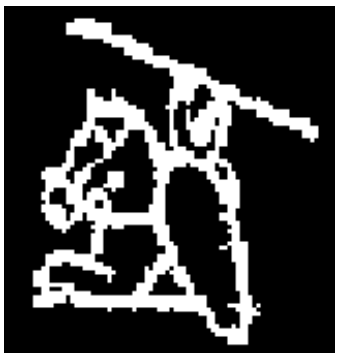

Original image

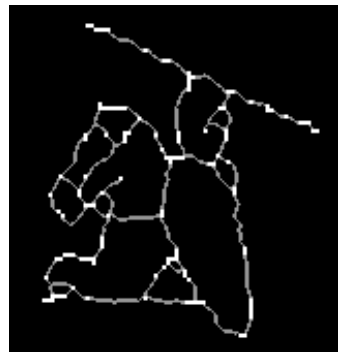

Obtained skeleton: the white pixels are the anchors points

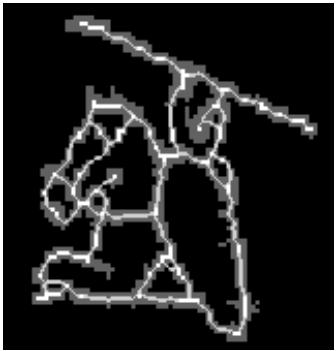

The skeleton merged into the original image

Fig. 1. Example of skeletonisation based on order independent thinnings and ultimate eroded set as anchor points.

method deletes all simple pixels, each simply connected component will be reduced to a single pixel. A first possibility to avoid this situation is to delete only specific configurations of simple pixels. This is usually done by considering a family of homotopic structuring elements. Consequently, the resulting skeleton depends on the chosen structuring elements, the order in which they are applied, and the order used for processing the image pixels.

Another approach is to first characterise a subset of pixels belonging to the skeleton called the anchor points Vin91Sch94. The anchor points are by definition nonsimple and cannot be deleted when thinning the image. By doing so, it is possible to thin the image with all possible homotopic structuring elements without reducing each simply connected set to a single pixel. However, the result still depends on the order used for processing the image pixels. This problem is eluded by considering order independent thinning. Some properties of the obtained skeleton directly depend on the chosen set of anchor pixels. A commonly used set of anchor points is composed of the centres of the maximal disks which guarantee that the obtained skeleton is suited for the reconstruction of the original image, but it is very sensitive to small boundary variations. Another possibility for the anchor points is to use the ultimate eroded set or, equivalently, the regional maxima of the distance function [Mey89]. In both cases, each $C C_{8}^{1}$ of the original image contains at least one anchor point, and at each step of the thinning algorithm the current image will have the same property. Thus we are sure that if $P$ is simple (and is not an anchor point), $C C_{8}^{1}(P)$ contains at least one anchor point (i.e., one nonsimple pixel). Thus if a pixel is in one of the configurations of subsection 3.4 we are sure that it is independent. This is an important issue because it allows us to skip the 8-deletability test and thus provides us with an algorithm in $O(n)$.

If the computation of the set of anchor points is independent of the pixels treatment and invariant through $\pi / 2$ rotations, then the resulting order independent thinning skeleton inherits these properties (e.g., when using the centres of maximal disks or ultimate eroded set). Figure[1 shows an example of skeletonisa- 
tion using ultimate eroded as anchors points. Notice that the obtained skeleton is not so thin as those obtained through other thinning approaches. Indeed, a single algorithm cannot simultaneously be independent of the order in which pixels are processed and lead to one-pixel thick result (incompatible properties).

\section{Conclusion and perspectives}

We have introduced a homotopic thinning approach which does not require to choose a family of homotopic structuring elements. Due to its independence with regard to the order in which pixels are processed, it allows one to skeletonise a binary figure without making any arbitrary choice. Our current research aims at studying order independent thinnings in the case of 3-D binary images.

\section{References}

Bun69. O. Buneman. A grammar for the topological analysis of plane figures. In B. Meltzer and D. Mitchie, editors, Machine Intelligence, volume 5, pages 383-393, Edinburgh, 1969.

CM91. J.-M. Chassery and A. Montanvert. Géométrie discrète en analyse d'images. Hermès, Paris, 1991.

Kon95. T. Kong. On topology preservation in 2-D and 3-D thinning. International Journal of Pattern Recognition and Artificial Intelligence, 9(5):813-844, 1995.

KR89. T. Kong and A. Rosenfeld. Digital topology: introduction and survey. Computer Vision, Graphics, and Image Processing, 48:357-393, 1989.

Lan78. C. Lantuéjoul. La squelettisation et son application aux mesures topologiques des mosaïques polycristallines. PhD thesis, Ecole des Mines de Paris, 1978.

Mey89. F. Meyer. Skeletons and perceptual graphs. Signal Processing, 16:335-363, 1989.

Ron86. C. Ronse. A topological characterization of thinning. Theoretical Computer Science, 43:31-41, 1986.

Ron88. C. Ronse. Minimal test patterns for connectivity preservation in parallel thinning algorithms for binary digital images. Discrete Applied Mathematics, 21:67-79, 1988.

Ros70. A. Rosenfeld. Connectivity in digital pictures. J. Assoc. Comp. Mach., 17(1):146-160, 1970.

Sch94. M. Schmitt. One pixel thick skeletons. In J. Serra and P. Soille, editors, Mathematical morphology and its applications to image processing, pages 257264. Kluwer Academic Publishers, 1994.

Ser82. J. Serra. Image analysis and mathematical morphology. Academic Press, London, 1982.

Vin91. L. Vincent. Efficient computation of various types of skeletons. In M. Loew, editor, Medical Imaging V: Image Processing, volume SPIE-1445, pages 297$311,1991$. 